\title{
Kernos
}

Revue internationale et pluridisciplinaire de religion grecque antique

$29 \mid 2016$

Varia

\section{Les Enfants de Cadmos. Le paysage religieux de la Phénicie hellénistique}

\section{Panagiotis P. lossif}

\section{QpenEdition \\ Journals}

Édition électronique

URL : http://journals.openedition.org/kernos/2440

DOI : 10.4000/kernos.2440

ISSN : 2034-7871

Éditeur

Centre international d'étude de la religion grecque antique

Édition imprimée

Date de publication : 1 octobre 2016

Pagination : 456-463

ISSN : 0776-3824

Référence électronique

Panagiotis P. Iossif, «Les Enfants de Cadmos. Le paysage religieux de la Phénicie hellénistique », Kernos [En ligne], 29 | 2016, mis en ligne le 01 octobre 2016, consulté le 18 novembre 2020. URL : http://journals.openedition.org/kernos/2440 ; DOI : https://doi.org/10.4000/kernos.2440

Ce document a été généré automatiquement le 18 novembre 2020.

Kernos 


\title{
Les Enfants de Cadmos. Le paysage religieux de la Phénicie hellénistique
}

\author{
Panagiotis P. lossif
}

\section{RÉFÉRENCE}

Corinne BONNET, Les Enfants de Cadmos. Le paysage religieux de la Phénicie hellénistique, Paris, De Boccard, 2015.1 vol. 15,5 × 24 cm, 606 p., 117 fig. (De l'archélogie à l'histoire). ISBN : 978-2-7018-0371-5.

1 Résumer et critiquer dans l'espace de quelques pages un livre aussi important que Les Enfants de Cadmos de Corinne Bonnet (C.B.) est une tâche compliquée mais très instructive. L'A. se définit d'emblée comme historienne (11), mais son ouvrage est beaucoup plus qu'un livre d'histoire, qui envisage les paysages religieux comme le fruit d'interactions multiples et intègre les dimensions politiques, sociales, économiques, comme le fit Nicole Loraux dans Les enfants d'Athéna. Si l'A. s'était donné comme objectif d'égaler le livre de Nicole Loraux, on peut déjà la féliciter, parce que le pari est largement gagné.

2 Le cœur de la démarche consiste à interroger le devenir des panthéons, des pratiques cultuelles, des mythes et formes de croyance au lendemain de la conquête des royaumes phéniciens par Alexandre. Pour ce faire, l'A. recourt à des outils anthropologiques, parfois aussi sociologiques, pour affirmer sa conviction qu'appliqués aux terrains " antiques", ils sont susceptibles de renouveler nos questionnements et nos grilles de lecture.

3 Deux éléments importants ressortent de cette enquête: 1) la césure entre ce qui précède et ce qui suit la conquête d'Alexandre ne doit pas être surévaluée, dans la mesure où d'importantes évolutions culturelles sont largement amorcées dès l'époque perse ; 2) les logiques d'interaction culturelle ne peuvent être ressaisies dans le cadre 
étroit de ce que l'on appelle une "acculturation " grecque, comme s'il s'agissait d'un processus unilatéral et presque mécanique. Ainsi, à la fin de son étude, C.B. permet de rejeter une fois pour toutes des termes comme "acculturation des orientaux", " hellénisation des dieux orientaux ", " orientalisation des dieux grecs », etc.

Son analyse est très influencée par les travaux menés par Marshall Sahlins sur le terrain hawaïen : tenant compte du fait que la culture grecque représente la modernité (comme le fit la culture anglaise-occidentale à Hawaï), de quelle manière les cités phéniciennes ont-elles réagi face à cette " modernité » ? Une analyse fine montre que le processus fut très compliqué : la réception, l'assimilation ou la domestication des nouveaux "produits ", loin d'affaiblir les cultures indigènes, les renforceraient et contribueraient même au développement des relations sociales traditionnelles.

Comme le sous-titre du livre l'indique, il s'agit d'une analyse de "paysage». Cette notion est envisagée sous l'angle religieux, des pratiques et des croyances, de cette construction artificielle qui s'offre devant nos yeux et fait appel à notre perception des modernes, parce que la notion de " paysage » est une catégorie moderne, absente de la mentalité des cultures qui sont en examen (cf. les travaux de François de Polignac pour les limites de cette notion, mais surtout pour les possibilités qu'elle offre à la recherche).

6 Ce livre vient également de combler un vide dans l'histoire phénicienne ; la récente synthèse de Julien Aliquot couvre la période romaine, quand les nombreuses études des Elayi traitent, en premier lieu, des périodes avant la conquête d'Alexandre (pas toujours sous le même angle religieux et méthodologique qu'Aliquot et Bonnet). Les rares sources qui peuvent être datées avec certitude de la période hellénistique, comme l'avait déjà signalé Fergus Millar, ne constituent pas une limite pour l'A., qui déploie une analyse exhaustive et détaillée de l'ensemble de la documentation disponible, faite des vas-et-viens dans le temps (et dans le paysage phénicien) pour "comparer l'incomparable", comme dirait Marcel Detienne, mais qui offre, surtout, un cadre méthodologique rigoureux suivi d'un questionnement pertinent et efficace.

7 Une notion fondamentale que l'A. introduit et qui guide son analyse est celle du Middle Ground développée par Richard White dans son étude des interactions entre populations des différentes cultures autour de la région des Grands Lacs entre 1650 et 1815. Cette notion désigne un espace animé de relations et de transactions menées par une pluralité d'acteurs poursuivant des buts variés qui s'agencent plus ou moins les uns aux autres. Elle oriente vers les espaces intermédiaires, vers l'entre-deux où intérêts et affiliations s'enchevêtrent pour donner naissance à un nouveau paysage culturel. Ce processus se caractérise par des négociations, des acclimatations; il est long et incertain.

Le livre se divise en quatre parties et neuf chapitres, suivis d'une longue conclusion. Le premier chapitre (Dans les pas d'Alexandre en Phénicie : la mémoire des vainqueurs, le silence des vaincus, p. 41-106) est le plus « historique » de tous. C'est le chapitre qui retrace le moment de la conquête de la province (avec des prolongements utiles et pertinents sur la conquête du reste de l'empire perse) par le Macédonien. L'A. met bien en évidence la partie unilatérale des sources qui retracent l'histoire, presque toutes d'origine grecque et qui adoptent, sans trop de questionnement, le point de vue du vainqueur sur les événements. L'analyse de C.B. met bien en exergue la volonté des historiens d'Alexandre de présenter la conquête comme une guerre défensive, une sorte de croisade légitime soutenue par les dieux, une notion tellement actuelle! L'analyse 
montre que la Phénicie n'est pas une étape secondaire dans le projet du Conquérant et que le siège de Tyr, auquel l'A. offre une longue place, en témoigne. L'épisode violent du siège de Tyr et de sa destruction est vu et décrit par l'A. sous un angle religieux : le tout commence par le refus des Tyriens de laisser Alexandre offrir un sacrifice à Héraclès, i.e. Melqart, dans son sanctuaire insulaire. Il est évident qu'en faisant ce geste, Alexandre se posait comme le successeur légitime des rois de la ville, qui tiraient leur légitimité de la filiation à Melqart. L'A. reprend cette idée du siège de Tyr comme une sorte de miroir de la guerre de Troie, où Alexandre est appelé à assumer le rôle d'un nouvel Achille. C.B. offre une très intéressante comparaison entre la conquête brutale de Tyr et la visite et l'accueil d'Alexandre à Jérusalem. Même si l'historicité de ce dernier épisode raconté par Flavius Josèphe est mise en doute, il n'est pas sans intérêt de noter que Yahvé semble réussir là où Melqart avait échoué : la providence divine du dieu des Juifs transforme Alexandre en allié (symmachos), quand Melqart en a fait un ennemi violent. Ce jeu de miroir religieux pour expliquer les opérations militaires est très utile parce qu'il met en exergue les discours identitaires, grecs et autres, qui se forment autour de la divinité tutélaire de la ville (ou de la dynastie). Le grand mérite de C.B. est d'écouter la version des vaincus, même si leur voix a disparu sous le poids des vainqueurs; elle nous offre des outils méthodologiques subtils pour bien cerner cette voix dont les échos méritent d'être écoutés.

9 La deuxième partie du livre, composée des chapitres deux à cinq (107-327), constitue une analyse de l'émergence des nouveaux paysages après la conquête macédonienne. C.B. suit un ordre géographique et commence par la ville phénicienne la plus septentrionale (Arados ; 109-152) pour terminer avec Tyr (269-327), tout en passant par Byblos (153-196) et Sidon (199-268). On se demande toutefois pour quelle raison, Bérytos, qui devrait, d'après le modèle analytique de l'A., se trouver entre Byblos et Sidon, ne fait pas partie de ce livre.

Dans chacun de ces chapitres, la présentation des sources et l'analyse suivent un modèle unitaire : l'A. commence par l'examen des sources relatives à la ville même et aux paysages religieux urbains, avant de s'interroger sur le rôle des sanctuaires extraurbains, souvent même des sanctuaires des confins. Arados est une ville important parce qu'elle était la seule ville phénicienne à faire partie du giron séleucide pendant tout le conflit qui opposa les derniers aux Lagides. Cette opposition, qui se déroule pendant les six Guerres de Syrie, aura la Phénicie comme scène principale et sera un élément formateur des paysages religieux et culturels des différentes cités. Pour Arados, C.B. montre très bien la transition depuis la royauté locale vers une tutelle grecque et les répercussions de ladite tutelle sur le plan symbolique et religieux. La royauté aradienne (comme les autres dynasties phéniciennes, avec les nuances nécessaires) incarnait le principe de la souveraineté divine, i.e. d'un roi représentant des dieux sur terre. Leur défaite par les Macédoniens donna un coup fatal à la royauté phénicienne et fut souvent interprétée comme une défaite des dieux royaux. C.B. analyse le paysage religieux avant l'arrivée des Grecs en se focalisant sur le sanctuaire "thérapeutique » d'Amrit, à $7 \mathrm{~km}$ au sud d'Arados qui abritait sans doute un culte à Eshmoun. Elle montre que l'aspect «thérapeutique » souvent attaché au sanctuaire et ses cultes est réducteur, dans la mesure où le site fut dédié à un Baal vigoureux, victorieux et protecteur et que l'aspect «thérapeutique " ne constitue qu'un aspect parmi d'autres. Le sanctuaire montre déjà un éclectisme de styles mêlant des éléments phéniciens, égyptiens, chypriotes, anatoliens ou grecs, qui se poursuivra après l'arrivée des Macédoniens. Particulièrement intéressante est la présence, à Arados, d'un 
gymnase dédié aux dieux traditionnels du gymnase grec (Hermès et Héraclès), dont la présence est connue par une base de statue bilingue (grec-phénicien). L'A. analyse subtilement la signification de cette dédicace bilingue dans la logique du middle ground pour en conclure sur le rôle d'une élite locale fortement impliquée dans la vie publique formée sur des institutions grecques. Un cas très important de ce chapitre est le sanctuaire de Baitocécé qui se trouvait dans la pérée de la ville. L'A. conclut de manière juste que l'hypothèse qui présente ce sanctuaire comme un sanctuaire confédéral n'est pas démontrable en l'état actuel de la documentation. C.B. offre une excellente analyse $\mathrm{du}$ célèbre dossier épigraphique trouvé sur l'enceinte du sanctuaire. Une seule objection à cette analyse : la lettre d'un roi «Antiochos " présente toute une série de problèmes épistolaires, ce qui laisserait supposer qu'on a affaire à un document pseudépigraphe fabriqué pour soutenir les prétentions du clergé local. Si cette hypothèse, que nous analyserons ailleurs, s'avère pertinente, la question de l'asylie du territoire devra se poser sous un prisme romain plutôt qu'hellénistique. Comme le chapitre se termine sur un excursus numismatique, on ne peut que féliciter l'A. de la place qu'elle accorde à ce médium tellement important pour la compréhension des cultes et des mythes. Souvent négligé ou utilisé à titre d'illustration par les historiens (et les historiens des religions), il trouve ici la place qu'il mérite. Quelques termes techniques échappent à l'A. (p. 115, le $\Gamma$ n'est pas une "légende» mais bien un « monogramme » ou un « symbole » qui, par ailleurs, ne semble pas faire référence au roi Gérostratos pour des raisons techniques), mais dans l'ensemble, les monnaies trouvent la position qu'elles méritent dans ce type d'étude.

11 Le chapitre suivant traite du paysage religieux de Byblos, la première ville au-delà de la frontière qui sépare les Séleucides des Lagides jusqu'à 199/8 av. J.-C. Byblos, ville créée par Kronos-El au début des temps, ville lagide qui entretient des rapports traditionnels avec l'Égypte, fut le site de la Dame de Byblos ou Baalat Gubal, que C.B. rapproche à juste titre de la déesse Hathor, et d'un dieu représentant de la puissance souveraine et performative. Ce couple est ici, comme ailleurs en Phénicie, lié à la famille royale locale. L'A. suit les aventures de la maîtresse de Byblos soumise à des dynamiques complexes et opère de fins rapprochements avec Astarté et surtout avec Isis; dans tous les cas, elle dresse le portrait d'une déesse souveraine résultant d'un dialogue continu entre les élites locales et les nouveaux maîtres, le domaine du middle ground. La divinité masculine de Byblos est celle d'Adonis. C.B. montre très clairement que cette divinité fut récupérée par les Lagides dans leur propagande aulique afin de renforcer leur capital symbolique dans une ville qui, d'une part, entretenait des liens millénaires avec l'Égypte et, d'autre part, constituait un point névralgique sur la frontière avec les Séleucides. Les dynamiques de ce processus, les assimilations ou rapprochements avec d'autres divinités portent une coloration égyptienne adossée d'éléments grecs et phéniciens, afin de créer une image polyvalente, «multi-représentative » sous un vêtement d'essence grecque. Encore une fois, la notion de middle ground s'avère féconde pour esquisser un paysage religieux phénicien souvent réduit à l'idée de l'hellénisation. La dernière partie de ce chapitre examine l'arrière-pays de Byblos (les sites d'Afqa, Yanouh et Maad), où l'A. observe les mêmes formes d'éclectisme religieux que celles des sites urbains.

12 Les mêmes dynamiques et paysages esquissés pour Arados et Byblos sont observés à Sidon. Le panthéon de cette ville est dominé par un couple divin souverain dont l'élément féminin est Astarté (présente dans les noms théophores des rois locaux). Pour C.B., le parèdre masculin doit être identifié à Eshmoun, le dieu bienfaisant et 
guérisseur, associé également à Melqart. L'A. argumente de manière convaincante que Sidon fut une ville "grecque " avant la conquête d'Alexandre : un Sidonien n'avait pas à choisir entre la Perse (force souveraine de la ville) et la Grèce (élément de culture dominant en Méditerranée orientale), mais il pouvait jouer dans cet espace intermédiaire aux limites floues, que la notion de middle ground explique de manière précise. Après la conquête, la ville fut dotée d'institutions grecques et d'une garnison de provenance ethnique variée. L'étude du sanctuaire extra-urbain d'Eshmoun à Bostan esh-Sheikh offre à l'A. un excellent cas d'étude pour déployer sa méthode et tirer des conclusions utiles. Ce sanctuaire dont on connaît quatre phases de construction témoigne de l'éclectisme phénicien tant dans le domaine religieux qu'architectural. La dite « tribune d'Eshmoun », les temple-boys (et girls), les trônes « vides » ou la « frise des enfants ", autant des monuments célèbres de l'art phénicien, sont examinés sous l'angle nouveau et prometteur du middle ground. Pour ce qui est des temple-boys, il faudra maintenant affronter la récente interprétation proposée par Claude Baurain, qui les lie (ceux de Chypre, mais le schéma pourrait fonctionner aussi pour Sidon) à Némée et au culte local d'Opheltès à travers un réseau complexe de généalogie divine de la dynastie royale ${ }^{1}$. D'autre part, la datation de la «tribune d'Eshmoun» est un élément majeur pour l'interprétation correcte du monument et sa mise en contexte. Tenant compte de l'importance de la présence séleucide sur le site (explicitement mise en exergue par les inscriptions rapportées par l'A. dans son dossier épigraphique), il ne nous semble pas impossible d'abaisser la date du monument au début du $\mathrm{II}^{\mathrm{e}} \mathrm{s}$. av. J.-C., juste au moment de la conquête de la région par Antiochos III. La comparaison stylistique avec les sarcophages historiées de Sidon n'offre pas un argument de datation suffisant et rien n'empêche, ni d'un point de vue historique, ni d'un point de vue archéologique, de dater la tribune $\mathrm{du} \mathrm{II}^{\mathrm{e}} \mathrm{s}$. av. J.-C. Nous avons montré récemment l'importance d'Apollon comme archégète des Séleucides ${ }^{2}$ et il semble plus prudent de placer l'introduction d'Apollon et son association à Eshmoun au moment de la conquête séleucide. Quoi qu'il en soit de ce point, C.B. montre de manière magistrale que le dieu tutélaire du sanctuaire ne fut pas simplement un dieu "thérapeutique », mais un dieu protecteur de l'enfance dans son ensemble, comme classe d'âge, celle qui assure la pérennité de la communauté. Ce chapitre est une véritable démonstration méthodologique indispensable à tout étudiant des polythéismes anciens, dont les dynamiques complexes et leur fluidité demandent des lectures subtiles loin de l'oversimplification de bon nombre d'études. Le paysage sidonien se complète par un excursus au sanctuaire de Kharayeb (sanctuaire rural dont la fonction se rapproche de celle de Bostan esh-Sheikh), une analyse de l'aspect maritime de l'Astarté locale et ses rapports avec Cos à travers le dossier épigraphique bilingue, et surtout par une analyse de l'inscription de Diotimos. Cette dernière montre les stratégies interculturelles des élites locales fortement «hellénisées ». Diotimos, un Sidonien qui assure la charge de dikastès, offre un intéressant discours identitaire à travers les parentés mythologiques qu'il établit entre Sidon et la Grèce. L'analyse de l'A. est inscrite dans cette même logique de middle ground et souligne la capacité des élites phéniciennes à utiliser la «boîte à outils " à leur disposition pour mieux établir leur position. Un élément supplémentaire qui pourrait ressortir de cette inscription est le rapport aux Jeux néméens : comme je l'ai déjà signalé, Claude Baurain propose d'associer les temple-boys à Opheltès, héros local de Némée ; or, l'inscription de Diotimos offre le lien direct entre Némée et Sidon, et pourrait servir dans la compréhension des temple-boys sous un angle 
néméen (mais ceci reste à explorer). C.B. referme son chapitre sur Sidon sur une brève référence aux stèles funéraires peintes de la nécropole ${ }^{3}$.

Le dernier chapitre de la deuxième partie traite de Tyr et reprend le fil là où le premier chapitre l'avait abandonné : au moment de la conquête par Alexandre. À Tyr, plus qu'ailleurs en Phénicie, le dieu tutélaire s'associe à la royauté locale, comme Melqart est le « roi de la ville». L'analyse du dossier épigraphique par C.B. montre que sous la domination des Lagides Melqart s'estompe sous la personne du roi (ou du couple royal). Nous ne sommes pas certain que «la tradition locale est sauve dans son noyau essentiel » (279) parce que Melqart est absent des documents officiels lagides, même sa possible interpretatio graeca, i. e. Héraclès, n'apparaît pas sur ces documents. Une présence « discrète » du dieu sous la personne du roi ne peut pas être suspectée dans l'état actuel de notre information. Il nous semble plus prudent d'assumer, contra l'A., que les Lagides n'ont pas fait appel à l'aspect royal du dieu de Tyr, parce que ceci ne servirait pas leur capital symbolique. L'A. a parfaitement démontré que les Ptolémées n'ont pas hésité à récupérer Adonis de Byblos afin de s'assurer la protection de la frontière avec les Séleucides; le dieu de Tyr ne peut, en aucun cas, être associé au couple royal alexandrin. Nous avons aussi quelques objections concernant la terminologie utilisée par l'A. pour les cultes royaux hellénistiques (à compléter maintenant pour les Séleucides par Iossif 2014). Le terme " assimilation » d'un roi à une divinité (285) est caduc et devrait être abandonné ${ }^{4}$. Le roi hellénistique (comme l'Empereur romain) ne s'assimile pas à une divinité, « Alexandre comme Zeus » est une absurdité pour citer Andrew Stewart ${ }^{5}$; toute association du royal avec le divin exprime la piété, l'eusebeia du roi envers un dieu, visuellement exprimée par les attributs divins et épigraphiquement par les épiclèses. Ainsi, Aphrodite Stratonikis à Smyrne n'est pas une forme d'assimilation entre la reine et la déesse, mais bien une manière d'exprimer la piété royale envers Aphrodite (de même à Iasos).

14 Le chapitre sur «Melqart et Héraclès » (290-304) offre l'occasion à l'A. de tester sa méthode et d'offrir une brillante démonstration de l'intégration d'une cité vaincue et humiliée dans le domaine et l'idéologie royaux en suivant l'approche du middle ground. Des sources variées sont passées en revue. Une petite objection: l'A. interprète le changement des types monétaires sur les monnaies tyriennes à partir de l'autonomie de la cité (126/5 av. J.-C.) comme une expression visuelle d'un changement de maître et le retour du dieu tutélaire ; elle a sans doute raison concernant le remplacement de la tête du roi séleucide par celle de Melqart. On ne peut en dire autant du maintien des types de revers : le fait que les monnaies autonomes conservent l'aigle posant ses pattes sur un éperon de navire et tenant une branche de palmier derrière son dos n'est pas une évocation $d u$ «renouveau escompté de l'antique puissance maritime et du leadership tyrien en Phénicie » (297-298). Nous avons récemment montré 6 que les types de revers sont typiquement lagides avec quelques références phéniciennes, mais en aucun cas tyriennes. Le maintien de ces types de revers aussi bien par les Séleucides, quand ils ont pris possession de la province après le règne d'Antiochos $\mathrm{V}$, que par les Tyriens après leur indépendance, répond à des besoins numismatiques et économiques plutôt qu'à une supposée expression de la primauté tyrienne sur la Phénicie. De plus, la dédicace grecque de l'autel du gymnase de Tyr doit être interprétée très prudemment: il s'agit d'une dédicace typiquement grecque que seul son contexte archéologique différencie des myriades d'autres du même type trouvées éparses dans le monde hellénistique. Toute conclusion se fondant sur un hypothétique rapprochement avec la bilingue du gymnase d'Arados doit être évité. Pour terminer ce chapitre, l'A. décortique 
le paysage religieux d'un autre sanctuaire des confins, celui d'Oumm el-Ammed. L'architecture des deux temples d'Astarté et de Milkashtart témoigne de ce goût pour l'éclectisme et « l'hybridation » déjà observé sur tous les sites en question. L'analyse de l'A. confirme un Milkashtart, Seigneur de Hammon, maitre des lieux sacrés, accompagné d'un messager divin. La conclusion de l'analyse de C.B. sur le rôle du dieu et du sanctuaire est probante: "Le culte de Milkashtart s'inscrit en tout cas dans un double registre de proximité et de distance par rapport à Tyr : Milkashtart est 'comme' Malqart, mais on a retiré de son nom l'élement qrt, qui renvoie clairement à Tyr " (321). Quelle meilleure démonstration de l'apport de l'étymologie doublée d'une analyse rigoureuse des données archéologiques et topographiques pour saisir un paysage religieux complexe et fluide !

15 La troisième partie (composée des deux chapitres ; 329-412) abandonne la description géographique et topique de la question pour adopter un point de vue transversal et tropique. En utilisant la notion charnière de middle ground, l'A. détermine des réseaux en utilisant la "théorie des réseaux", cette méthode si souvent utilisé, et dont on a abusé souvent, dans la recherche récente. C.B. offre un modèle méthodologique pour l'utilisation de la méthode dans le domaine de l'histoire des religions. Dans un premier temps (331-365), l'A. dresse subtilement et avec patience les généalogies et les parentés divines des familles légendaires de Phénicie avec le monde grec. D’abord, les péripéties d'Agénor, ensuite celles de Cadmos. L'A. témoigne d'une maîtrise impressionnante des innombrables variations de ces mythes et les explique de manière claire et concise. La notion de la syngeneia est mise en exergue comme outil diplomatique, mais surtout comme moyen nécessaire pour naviguer dans le domaine flou du middle ground créé entre les Grecs et les Phéniciens, et où le mythe est utilisé comme arrière-plan pour l'établissement des réseaux religieux (et rituels). L'inscription de Diotimos, analysée dans son large contexte de la religion de l'époque hellénistique, montre que les Phéniciens (ou Sidoniens, les deux termes étant interchangeables dans la grammateia grecque) maîtrisaient parfaitement le langage symbolique de l'époque et les stratégies nécessaires pour se mesurer aux Grecs afin d'affirmer leur propre valeur, voire de témoigner de la prééminence de leur traditions mythologiques par rapport à celles des Grecs. L'A. rend cette conclusion incontestable, tout en déployant les éléments identitaires de ce qui fait d'un individu de l'époque hellénistique, un homme de son temps, un homo hellenisticus. Le chapitre intitulé « De l'Arcadie en Phénicie ?» (354-360) offre avant toute autre chose les éléments conceptuels pour saisir la manière dont les Grecs interprétaient les nouveaux territoires, comment ils récupéraient des terres nouvellement conquises et construisaient des paysages symboliques et imaginaires qui étaient des reflets de la Grèce. Récemment, Laurent Capdetrey et Paul Kosmin ont œuvré dans cette direction pour ce qui est du territoire séleucide, mais sans pour autant faire appel à l'aspect religieux si finement appréhendé par C.B.

Le présent lecteur, archéologue et spécialiste de l'iconographie, ne peut que saluer le dernier chapitre de la troisième partie du livre (367-411), qui porte sur les stratégies iconographiques pour récréer les nouveaux paysages religieux. La qualité de l'analyse nous oblige de paraphraser Georges Clemenceau et dire que "l'iconographie! C'est une chose trop grave pour la laisser à des archéologues ». C.B. prouve que le regard d'une historienne (des religions) apporte des éléments neufs et frais. Que retenir en premier? Le fait qu'elle fait connaître dans le monde académique francophone les importants travaux de Marshall McLuhan qui insiste sur le rôle du medium en tant que message (pas seulement en tant que véhicule du message)? L'analyse pertinente des quatre 
sarcophages de Sidon dans leur contexte religieux? L'identification de l'éclectisme et de l'hybridation des styles sur des media différents? Le fait que la distance entre un centre supposé et une périphérie supposée n'affecte pas vraiment les contrastes stylistiques et conceptuels? Ou bien le besoin de définir avec précision les notions que nous utilisons, telles que l'aniconisme? Les considérations numismatiques sont bienvenues dans ce domaine, même si les conclusions de l'A. ne sont pas toujours en accord avec les nôtres (cf. Iossif, in AJN 2014). Ceci dit, il nous semble que l'A. confond les notions de frappe monétaire et d'autonomie (symbolique ou réelle). La frappe de la monnaie n'est pas à elle seule une indication d'autonomie et les frappes dites quasiautonomes introduites par Antiochos IV dans une série de villes de la partie orientale du royaume séleucide ne peuvent en aucun cas être prises comme des marques de faiblesse d'une autorité royale. Les types monétaires répondent très souvent à des impératifs économiques, financiers, voire numismatiques, et les conclusions qu'on tire de leur utilisation doivent prendre en considérations ces aspects (de même que les volumes des émissions).

(4) deux dossiers particuliers : celui d'Athènes, du Pirée pour en être plus précis, et celui de Délos. C.B. offre une analyse exhaustive des sources relatives à la présence des Phéniciens dans ces villes grecques. Elle esquisse le rôle des Phéniciens dans plusieurs réseaux « internationaux » et " ethniques »; Athènes et Délos sont considérées comme des middle grounds pour ces Phéniciens qui négocient habilement leurs positions sur des terres nouvelles tout en restant fidèles à un lien (varié) avec leur patrie d'origine qui s'exprime à travers les coutumes, les dieux ou la langue.

18 L'étude est complétée par un épilogue, une longue bibliographie (mais qui ne reprend pas l'ensemble de références mentionnées dans le texte et qui nécessite une certaine mise à jour dans différents aspects) et un index thématique très utile.

19 Cette étude est fondamentale et fera date. Ce n'est pas un livre facile à lire, tant les informations, les analyses, les idées bombardent le lecteur de tous les côtés, et l'on referme le livre avec ce mal de tête bienvenu parce qu'il ne laisse pas l'esprit se reposer tant il est stimulant. J'ose même affirmer que l'A. dépasse les qualités Des enfants d'Athèna, tant son approche méthodologique est stimulante, ses analyses fines, les sources étudiées de manière minutieuse. Une grande qualité de ce livre, qui le distingue clairement du reste de la bibliographie francophone, est la maitrise des notions anthropologiques anglo-saxonnes tellement utiles, tellement... thought-provoking pour reprendre les nombreux anglicismes utilisés tout au long de ce magnifique livre. Néanmoins, on ne peut pas passer sous silence certaines faiblesses de l'édition, les coquilles trop nombreuses, une mise en page qui élimine parfois les espaces entre les mots ou change de police sans raison évidente. Je signalerai juste deux points qui rendent la lecture problématique pour le lecteur non averti : pages 69-70, l'A. décrit un Alexandre mais la figure 4 illustre une monnaie de Ptolémée $\mathrm{I}^{\mathrm{er}}$ portant, certes, l'image d'Alexandre, mais ne correspondant pas à la description offerte dans le texte. Aussi, on ne peut que regretter le fait que certaines sources iconographiques ne soient pas illustrées, en dépit des descriptions détaillées qui en sont données, surtout en considérant la très riche illustration de ce livre, avec la majorité des illustrations en couleur (p. 118-119: description du monnayage arwadite avant l'arrivée des Grecs; p. 358 : bas-relief de l'université américaine de Beyrouth). 


\section{NOTES}

1. .Cl. BAURAIN, «La contribution des Teucrides aux cultes royaux de l'époque hellénistique », in P.P. IOSSIF et al. (éd.), More than Men, Less than Gods. Studies on Royal Cult and Emperor Worship, Leuven, 2011, p. 121-155, en particulier p. 144-148.

2. .P.P. IOSSIF, « Apollo Toxtes and the Seleukids: Comme un air de famille », in IOSSIF et al., ibid., p. 229291.

3. .Récemment (P.P. IOSSIF, « Les 'cornes' des Séleucides : vers une divinisation 'discrète' ", CEA 49 [2012], p. 45-150, en particulier p. 107-110) nous avons repris l'analyse de ces stèles sous l'angle de leur armement et de leurs origines diverses et nous avons montré qu'il serait prudent de les dater de la phase lagide de la ville puisque la composition ethnique des défunts corresponde à celle des possessions lagides extérieurs à l'Égypte avant 198.

4. .P.P. IOSSIF, « The Charisma of the King. The 'discreet' association of the Ptolemaic and Seleucid kings with the divine", in A. DAVID et al. (éd.), Picturing Royal Charisma in the Near East (3rd millenium BC to $1700 A D$ ), Proceedings of the conference, Jerusalem 12-14 January 2015, Jerusalem, 2016, p. 84-117.

5. .A. STEWART, Faces of Power. Alexander's Image and Hellenistic Politics, Berkeley, 1993, p. 194, n. 10.

6. .P.P. IOSSIF, « Seleucid 'Eagles' from Tyre and Sidon: preliminary results of a die-study ", in N. HOLMES (éd.), Proceedings of the XIVth International Numismatic Congress, Glasgow 2009, Glasgow, 2011, p. 213-229 ; P.P. IOSSIF, «The Last Seleucids in Phoenicia: Juggling between civic and royal identity », AJN 26 (2014), p. 61-87.

\section{AUTEURS}

PANAGIOTIS P. IOSSIF

École belge d'Athènes 